The Egyptian International Journal of Engineering Sciences \& Technology, Vol 9, No 1 (2005)

\title{
FRACTAL NEURAL PROCESSOR
}

AbdelRazek AbouEINoor, A ALI FAHMY, ABDEILATIEF H. ABOUali, SABRY M. ABDELMOETTY

\author{
Abstract \\ In this paper, a novel fractal neural \\ processor is presented. The processor puts \\ the fractal image compression / \\ decompression to the speed of VLSI \\ realization. The processor is composed of \\ two modules, compression, and \\ decompression. The two modules operate \\ in ftill duplex mode. The compression \\ module operates, in two basic modes: \\ image learning and compressed image \\ production. During image learning, the \\ image is presented to the processor for \\ learning and storing the image domains in \\ an associative memory based on \\ classification. During die compressed \\ image production mode, the image is \\ presented to the processor to recall the best \\ match domain blocks and produce the \\ fractal parameter(s). The decompression \\ module starts with an initial state of the \\ image memory, normally zeros, and it \\ keeps evolving the image
}

